\title{
Creating an Atlas of the Anthropocene
}

\author{
Benjamin D. Hennig a, * \\ ${ }^{a}$ University of Iceland, ben@hi.is \\ * Corresponding author
}

Keywords: Cartograms, Atlas, Anthropocene

\begin{abstract}
:
The Anthropocene is a new geologic era, in which the effects of the human population influence the natural environment as much as the natural environment previously determined the existence of human life. The Anthropocene has become a vivid yet informal metaphor of global environmental change. In a broader context, global change contains the full range of human-environment relationships.

The debate about the complex relations and the diverse natures of the different spheres in which globalisation and global environmental change take place had an impact on how space is perceived in geography. These sometimes theoretical and often very complex interrelations make it necessary to find new representations in cartographic form.

In the past, map projections did not only solve specific challenges of navigation, such as the Mercator projection which helped to allow navigation over the world's oceans to become much easier and persists until today for its capability of making navigational tasks easier, also in modern computer algorithms. They sometimes also changed people's perception of the planet. The Gall-Peters projection is an example for how map projections were used to challenge the notion of power and question culturally biased or bigoted world views. It is based on the concept of a cylindric equal area projection in which every continent and every country is shown in its true physical size, but appears deformed in horizontal dimensions towards the higher latitudes and in vertical dimensions towards the Equator. While being controversial at the time in the 1970s, his map was acclaimed as a fairer view to tackle the imbalance between the wealthier North and poorer South of the world, an issue which was becoming ever more relevant and pressing at the time.
\end{abstract}

A different way of challenging people's views of our world is the use of cartograms. One central trait of cartograms is that of changing people's perception of the planet by applying the principle that the visual perception of a topic can support an immediate understanding of the underlying data, and that visualisation can turn complex data into easier-tounderstand representations. These allow a much different interpretation of the information that is shown compared to looking at mere numbers. For data with a geospatial background, a cartographic depiction such as a map thus remains a very obvious choice to visualise quantitative data. A cartogram transformation is a valuable alternative to make quantitative dimensions better understandable, but also to challenge the map-reader and provoke a response.

Using a novel gridded cartogram approach makes cartograms equally detailed and versatile as other map projections, by being based on neutral spatial units (rather than arbitrary administrative areas as in previous cartograms), being scalable, and allowing other geographic information being shown within the transformed map. Using earthquake risk as an example, a gridded population cartogram projection helps to understand earthquake risk zones related to the distribution of people on this planet and thus where this natural event turns into an actual hazard (Figure 1).

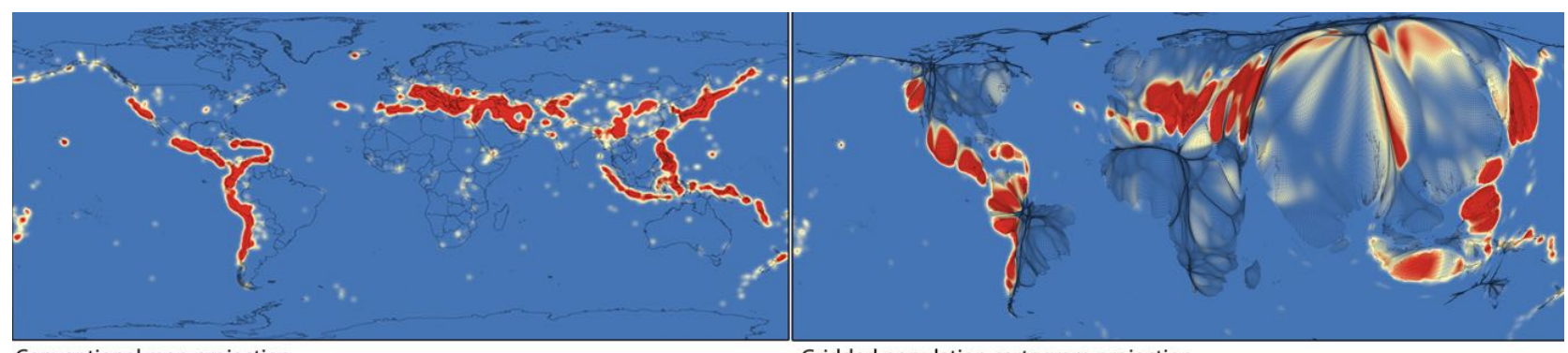

Conventional map projection

Gridded population cartogram projection

Figure 1. Cartographic visualisations of global earthquake risk zones using different map projections.

Gridded cartograms are therefore the first real map projection based on the concept of cartogram depictions. By using concepts from both cartograms and conventional map projections, they allow the diverse human and physical spaces to be made visible from a new perspective that no longer only uses land area as the main defining element of a map image.

The creation of an Atlas of the Anthropocene constitutes the first comprehensive effort to depict the complex humanenvironmental relations in novel thought-provoking ways. By utilising the gridded cartogram technique, the way humans 
live on this planet in their diverse social and natural environments will be shown in order to make the reader rediscover what it means to live on this increasingly 'human' planet.

While map projections often try to find the best solution for translating the third dimension into a flat depiction of the earth, a focus on the cartographic display of the social spaces looks beyond the constrained chains of the physical space and can contribute to new insights and understandings of the anthroposphere of the planet.

While humans are in the centre of the maps included in this atlas, the range of maps included do not solely focus on humans and human-environmental relationships, but also provide a new perspective of the multitude of spaces that characterise the natural environment that we live in, live from, interactive with, and most importantly have an impact on. Therefore the range of themes covers all spaces ranging from land, air and sea, looks at human as much as it does on animals, gives a new understanding of processes of globalisation, and shows both human inhabited and uninhabited areas to make us see the world anew.

In this presentation ongoing work on a new Atlas of the Anthropocene will be introduced. The goal of this atlas project is to improve the readers' understanding of the world during a time of drastic environmental change (Figure 2), just as Gerard Mercator depicted the world that eventually enabled a period of globalisation five centuries ago and Arno Peters helped us to comprehend the divided world of fifty years ago.

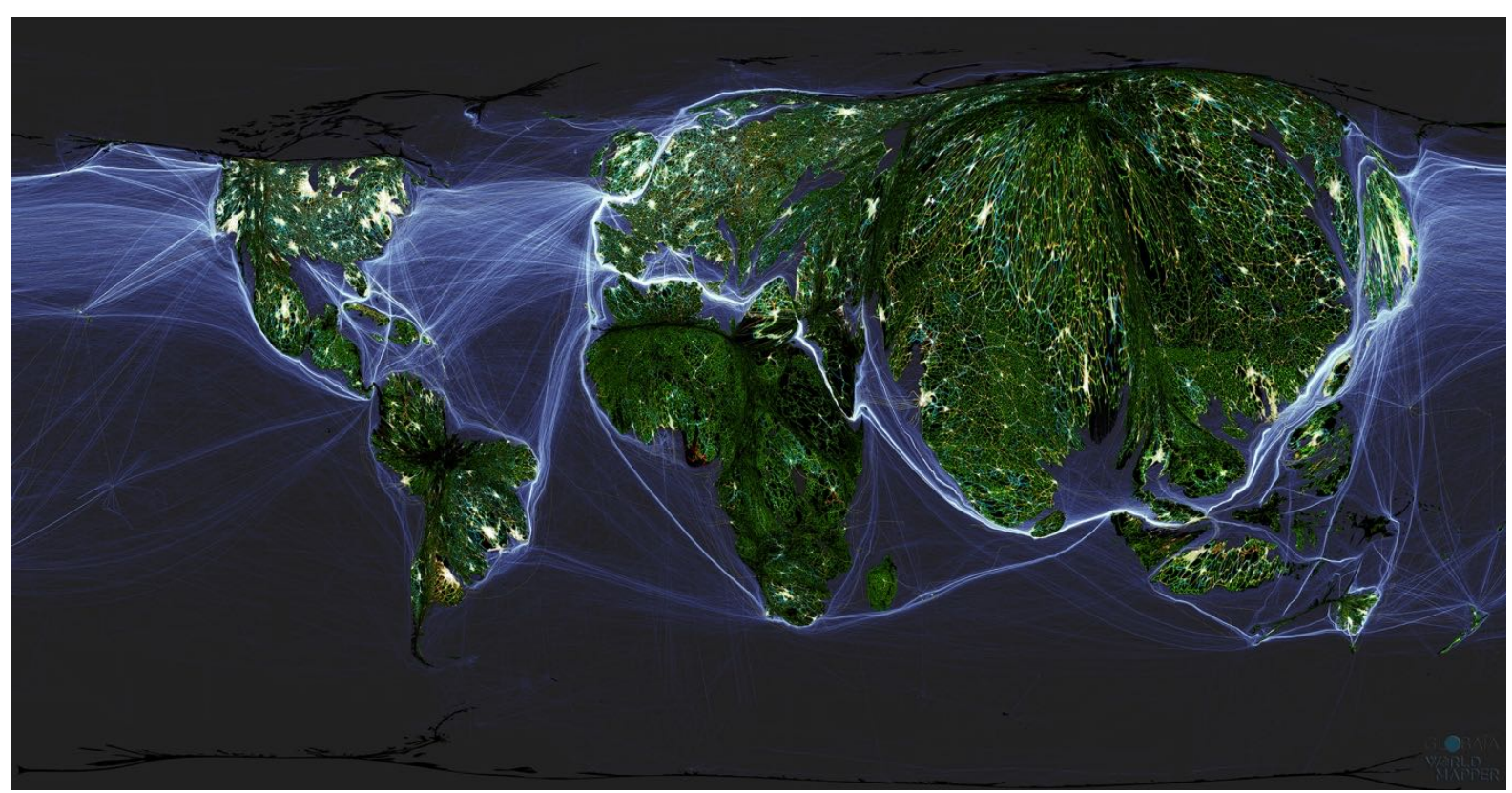

Figure 2. A gridded population cartogram showing key indicators of human impact on the planet. 